\title{
The role of anatomical anomalies in anterior communicating artery aneurysm rupture
}

Discipline of anatomy, Department I - preclinical disciplines, Faculty of medicine, University "Ovidius" Constanţa

Background and purpose: limited data exist to guide proper patient selection for preventive treatment of unruptured cerebral aneurysms. Cerebral aneurysms have been associated with anomalies of arterial segments that are forming the brain arterial circle of Willis but whether this association is also related to aneurysm rupture is not known. The occurrence of cerebral aneurysm rupture when a circle of Willis anomaly was present or absent was compared.

Material and methods: we have performed this study on a number of 312 cases, of which 87 were dissections, 22 dissection followed by plastic injection, 135 magnetic resonance angiography (MRA), 75 computer tomography angiography (CTA), 40 digital subtraction angiographies (DSA), 30 in vivo (intraoperatory) observation.

Brain vascular imaging was reviewed for aneurysm size, morphology and presence of anterior cerebral artery anomalies.

Results: we divided the study group in 2 cohorts, one control group of 272 cases, in which we have study the anatomical variants occurrence and aneurysm occurrence in general population and another included 45 patients admitted thru emergency room for subarachnoid hemorrhage, of those 38 were ruptured aneurysm of anterior communicating artery (ACoA). Mean aneurysm size was $8.9 \mathrm{~mm}$. An anterior cerebral artery anomaly was identified in 31 cases $(81.5 \%$ ). Ionescu C.

Department of Anatomy, Faculty of medicine, University "Ovidius" Constanţa, Romania

Aleea Universitatii, Nr. 1, Campus B

Constanţa, Romania

costin@anatomie.ro
Multivariate analysis revealed a higher risk of aneurysm rupture when an anterior cerebral artery was present. Conclusions: this study shows that anterior cerebral artery anomalies are more commonly found in ruptured as opposed to unruptured ACoA aneurysms. The presence of an ACA anomaly may be an important characteristic for selecting patients for preventive aneurysm treatment.

Keywords: aneurysm, rupture, ACA, asymmetry, hypoplasia, agenesis

\section{Introduction}

Preventive treatment of aneurysms via either clipping or coiling, as shown in the International Study of Unruptured Intracranial Aneurysms (ISUIA), was associated with an overall morbidity and mortality of approximately $10 \%$ at 1 year [1]. Aneurysmal subarachnoid hemorrhage however, is a leading cause of stroke disability and death in young patients, with an estimated initial mortality rate of $50 \%$ and up to $50 \%$ morbidity in survivors [2]. Rupture is not a common event though, with 51 (3\%) of the 1692 patients with cerebral aneurysms developing a confirmed aneurysmal rupture during follow-up in the ISUIA study [1]. Therefore, identifying risk factors for aneurysm rupture is fundamental in selecting patients who may truly benefit from preventive 
treatment. Current methods to risk stratify patients with unruptured cerebral aneurysms are based on a limited understanding of its natural history. Published risk factors for aneurysm rupture have been largely limited to demographic factors and rudimentary anatomic factors, including female gender, a history of smoking and/or hypertension, family history, as well as aneurysm dome diameter and location in the posterior circulation $[1,3]$. The paucity of detailed imaging based, quantifiable risk factors has led to considerable controversy about appropriate patient selection for preventive treatment. One example is the discrepancy in the literature on the risk of hemorrhage of aneurysms that are $7 \mathrm{~mm}$ or less in diameter. ISUIA concluded that aneurysms less than $7 \mathrm{~mm}$ without a history of subarachnoid hemorrhage located in the anterior circulation have zero risk of subarachnoid hemorrhage at 5 years [1]. In contrast, several series of exclusively ruptured aneurysms demonstrate an overwhelming majority to have diameters of less than $10 \mathrm{~mm}$ [4].

Circle of Willis variations, or a configuration that is not completely symmetric, is found in up to $40 \%$ of the population [5] and often includes a dominant anterior cerebral artery with a hypoplastic or absent contralateral anterior cerebral artery [6]. Tanaka et al demonstrated in 125 subjects, using two-dimensional cine phase contrast MRI, that flow rates in both the carotid and basilar arteries were significantly different with the presence of a circle of Willis variation [7].

\section{Material and Methods}

We have performed this study on a number of 312 cases, of which 272 cases were studied material for anatomical model as control group, it was of 87 cases of dissection, brains formalin treated 61 , 26 dissected fresh brains of these, only 22 cases we performed plastic injection, 20 cases by digital subtraction angiographies, 40 CT angiographies, 125 angiographies RM, they were made for other pathologies than aneurysm or subarachnoid hemorrhage (SAH). Another separate group studied consisted of 45 patients who had confirmed neurosurgical vascular pathology affecting the cerebral circulation, as first step they were investigated by standard CT examination to detect possible blood accumulation followed by CTA examination in 35 cases or MRA in 10 cases and / or digital subtraction angiography (DSA) in 20 patients, 30 of the patients underwent surgery for aneurysm suppression so cerebral arterial system could be evaluated prior to and during surgery.

Vessel diameter was measured perpendicular to the direction of the vessel and aneurysm size was measured at the greatest diameter.

Some CTA and MRA examinations were performed in the clinic Medimar Imaging Services Constanta. Cerebral angiography and intraoperative images were obtained courtesy of radiology and neurosurgical departments in Emergency Hospital "Prof. Dr. N. Oblu", "Science and Emergency Hospital Bagdasar - Arseni”, Bucharest.

\section{Results}

In control group we have study the anatomy of ACA, of which the caliber we have considered to be of great importance in local hemodynamic.

The gauge of ACA was variable, with a value between $0.3 \mathrm{~mm}$ and $3.8 \mathrm{~mm}$ on both sides, on the right side with values between $0.3 \mathrm{~mm}$ and $3.5 \mathrm{~mm}$ with a mean of $1.82 \mathrm{~mm}$ the left side with values ranging between $0.2 \mathrm{~mm}$ and $3.8 \mathrm{~mm}$ with a mean of $1.91 \mathrm{~mm}$.

A2 segment size differences right / left, they tend to disappear no longer a significant difference to the value of both relative and absolute. The junction of the two vascular axes thru anterior communicating artery the major caliber asymmetries disappear the ACoA being responsible for blood flow redistribution and so it's increased the pressure stress on the arterial walls. 
We have encountered a right-left A1 arterial segment asymmetry in gauge, the most commonly, in 48 cases, $17.6 \%$, the left A1 segment has an increased diameter and in 29 cases, $10.6 \%$, the dominant vessel was right ACA. We have considered hypoplasia when an arterial segment has a value less than $1 \mathrm{~mm}$. we observed a right A1 hypoplasia (Figure 1) in26 cases. 9.5\% and in 10 cases, $3.6 \%$ a left $\mathrm{A} 1$ segment hypoplasia (Figure 2).

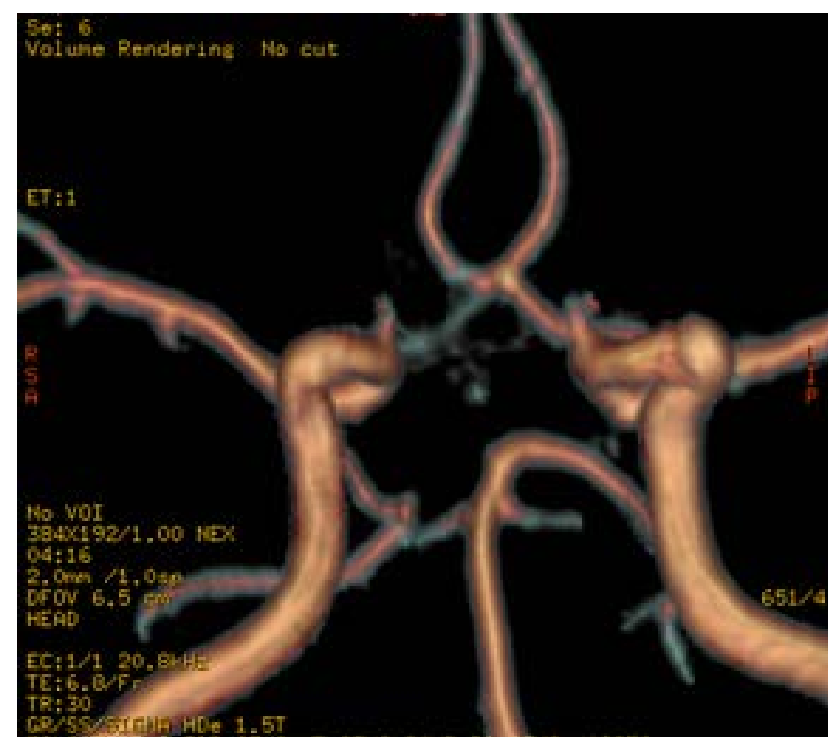

Figure 1 - Right Al hypoplasia

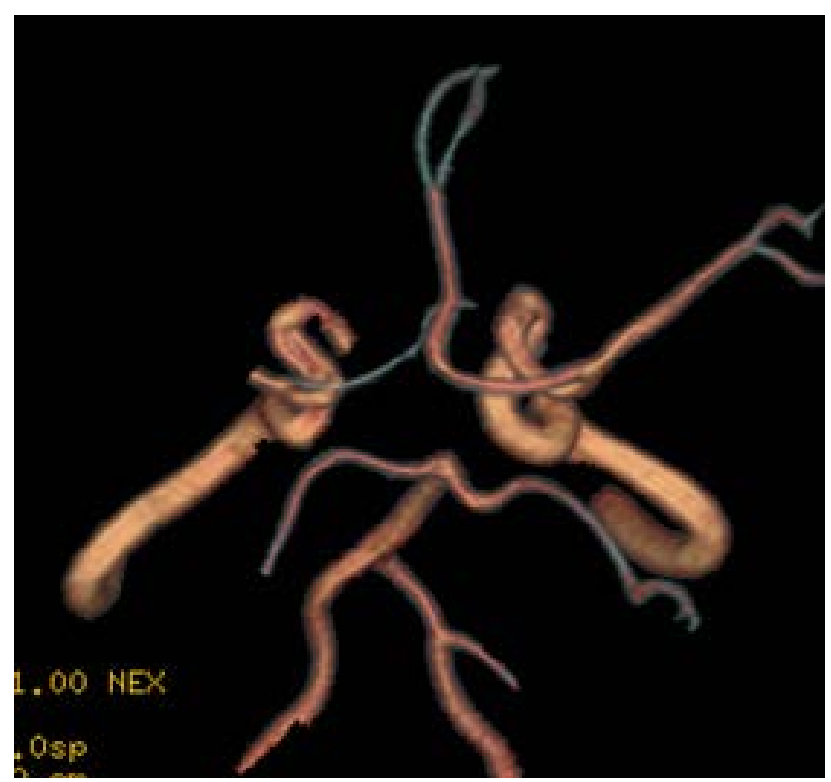

Figure 2 - Left Al hypoplasia
Regarding anterior communicating artery anatomical anomalies, we have encountered I found its caliber between 0.3 to $3.6 \mathrm{~mm}$, with a mean of $1.39 \mathrm{~mm}$. We found only in 64 cases its size less than $1 \mathrm{~mm}$, hypoplastic $(23.5 \%)$. We found only 29 cases double anterior communicating artery $(10.6 \%)$. In this case, the second communicating artery having a length of $0.5-4 \mathrm{~mm}$, size of between 0.4 and $1.8 \mathrm{~mm}$ (in 19 cases are hypoplastic, $7 \%$ ). In the other 7 cases, $2.6 \%$ anterior communicating artery bifurcates, with a common core abuse anterior cerebral artery and the branches will anastomose with opposite ACA, in five cases were triple, $1.8 \%$, in 4 cases had network appearance, $1.4 \%$. In 11 cases absent, 4\%, being either a side-to-side anastomosis in four of them, $1.4 \%$, or form a single artery azygos ACA in 4 cases or absence of lack of anastomosis between the two ACA, 3 cases $1.1 \%$.

In 6 cases we encountered a core of pericallosal arteries that are rising from ACoA, sually at origin this artery has a small infundibular dilatation, leading in time to the development of an aneurysm.

Ofthe cases included in the control group without subarachnoid hemorrhage or history of, I encountered 18 cases, $6.6 \%$ have identified the intracranial artery aneurysms. Of the 10 cases studied were anterior communicating artery aneurysms, representing $55.6 \%$, some of them developed at pericallosal artery origin from anterior communicating artery. in 4 cases, $22.2 \%$ have met middle cerebral artery aneurysms in 3 cases, $16.6 \%$ have found dilatation of internal carotid artery aneurysm in two anterior cerebral artery, one of which, 5.5\% in A3 segment the associate and azygos cerebral artery and one case $5.5 \%$, where I met the posterior communicating artery aneurysm in the cavernous segment of it. 


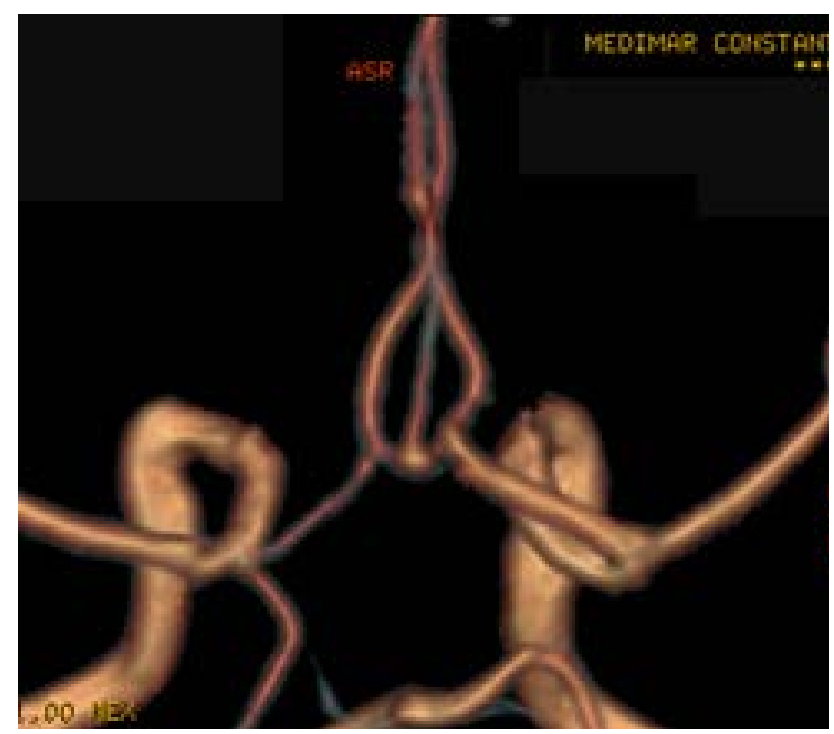

Figure 3-ACoA aneurysm at pericallosa origin

In terms of size the majority were mediumsized aneurysms between $7 \mathrm{~mm}$ and $17 \mathrm{~mm}, 13$ cazuri, $72.2 \%, 4$ cases, $22.2 \%$ of small-sized aneurysms under $7 \mathrm{~mm}, 211.1 \%$ of large aneurysms between 18 and $25 \mathrm{~mm}$, and only one, $5.5 \%$ more than $25 \mathrm{~mm}$. It was a slight female dominance of 18 cases 11 were in females and 7 in males.

In the study of the patients with subarachnoid hemorrhage, and aneurismal pathology confirmed, I tried to emphasize some specific features of the local anatomy. There is also a slight female predominance practically insignificant, Of 38 cases 20 were females $53 \%$, and 18 were males, $47 \%$.

In terms of size they were of small aneurysms of $6,15.8 \%$, medium sized aneurysms in number of $30,78.9 \%$ and large aneurysms in number 3, 7.9\% (Figure 4).

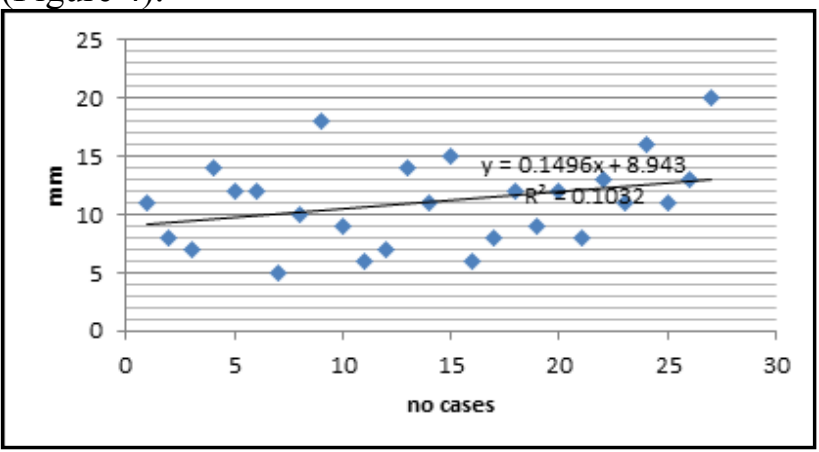

Figure 4-ACoA aneurysm size distribution
Regarding local anatomy anomalies, the results were surprising, because in most cases there is a deviation from normal morphology of anterior cerebrovascular system as: agenesis of segment A1 we found one case, $2.6 \%$ of the right and one case on the left, $2.6 \%$, in 9 cases, $23.7 \%$ found the right A1 segment hypoplasia in 5 cases we found hypoplasia of the left A1 segment in 14 cases there was a size mismatch between segments A1 with a dominant left side in 9 cases $23.7 \%$, a dominant right side in 5 cases $13.15 \%$, remaining 7 cases without abnormalities of symmetry A1 segments of the cerebral arteries. In these cases, in one case we found the presence of an arterial trunk pericalosal which rise from anterior communicating artery in a case anterior communicating artery bifurcates and in one case, $2.6 \%$ was an anterior communicating artery with network aspect with formation of 2 aneurysm at the origin of segments A2 (Figure 5)

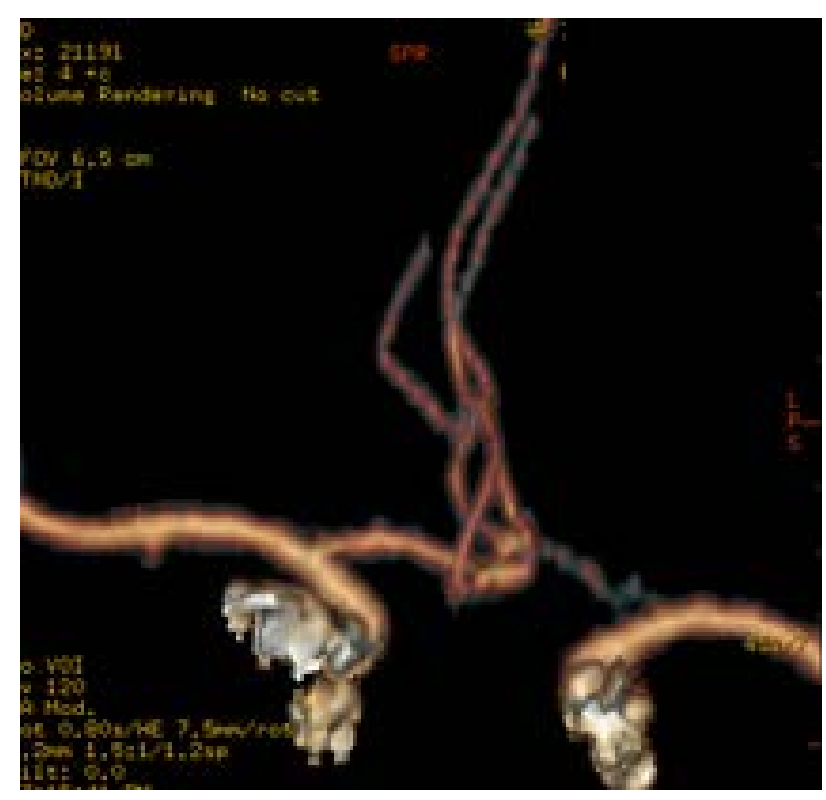

Figure 5-Network-like ACoA with 2 aneurysms 


\section{Discussion}

First described by Thomas Willis (1621-1675), an English physician, in his 1664 work De Anatomie Cerebri, the Arterial circle of Willis provides for vascular redundancy at the skull base that optimizes blood flow to the brain. This vessel structure enables interhemispheric flow through the $\mathrm{ACoA}$ and in two directions through both PCoAs in the event of diminished blood supply through one of these vessels. Flow through these collateral pathways that comprise the circle of Willis deviate from geometric optimality principles in that certain bifurcation, in particular the ACoA and PCoA, do not achieve minimal energy expenditure [8].

It has been observed clinically that most cerebral aneurysms are located in areas subjected to increased hemodynamic forces that is, at the apex of arterial bifurcations or on the curve of tortuous vessels rather than along straight vessel segments [9]. This may explain the remarkable predictability with regard to aneurysm location despite the wide

The two most common aneurysm locations within the circle of Willis are at the ACoA and the PCoA [12]. Bor et al demonstrated the putative role of hemodynamics in cerebral aneurysm formation in evaluating the CTA or MRA of 26 patients who developed de novo aneurysms after follow-up of normal cerebral vessels compared with 78 controls who did not develop an aneurysm [10].

Circle of Willis anomalies may accentuate these aneurysm inducing hemodynamic forces with greater flow at specific bifurcations to compensate for an absent or hypoplastic vessel elsewhere in the circle of Willis [6].

Considering the maximum diameter of the aneurysms, we have observed that in ruptured ones is a dominance of medium size aneurysm in between $7 \mathrm{~mm}$ and $17 \mathrm{~mm}$ (Figure 6).

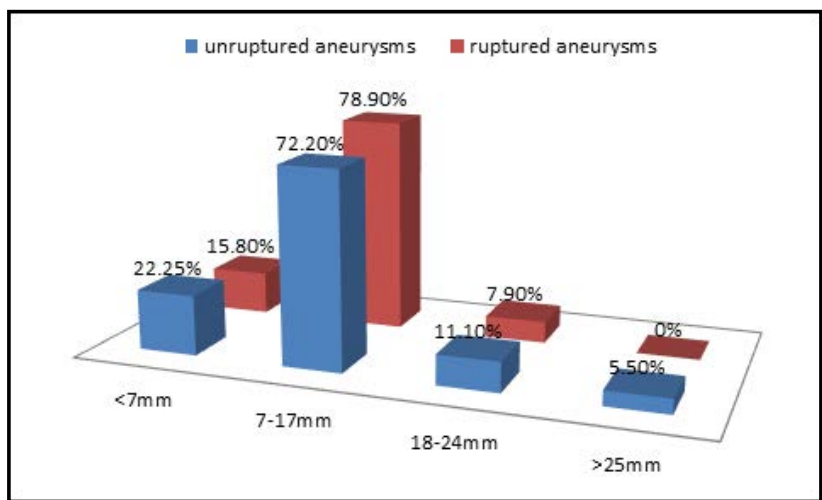

Figure 6-ACoA aneurysm size distribution compared ruptured/ unruptured.

Also we observed a dominance of female gender in ruptured aneurysm similarly with control group, meaning that the gender has no relevance in risk prognosis for rupture.

Our series demonstrates that, among ACoA aneurysms, circle of Willis anomalies occur more frequently in patients with ruptured cerebral aneurysms than in those that have not ruptured. This finding suggests that the presence of a circle of Willis anomaly in association with an ACoA aneurysm may be a risk factor for rupture.

Comparing the subarachnoid hemorrhage patients group with control group, we have observed, in case of ruptured, the predominance of A1 segments asymmetry, which includes agenesis, hypoplasia, and gauge asymmetry. (Figure 7)

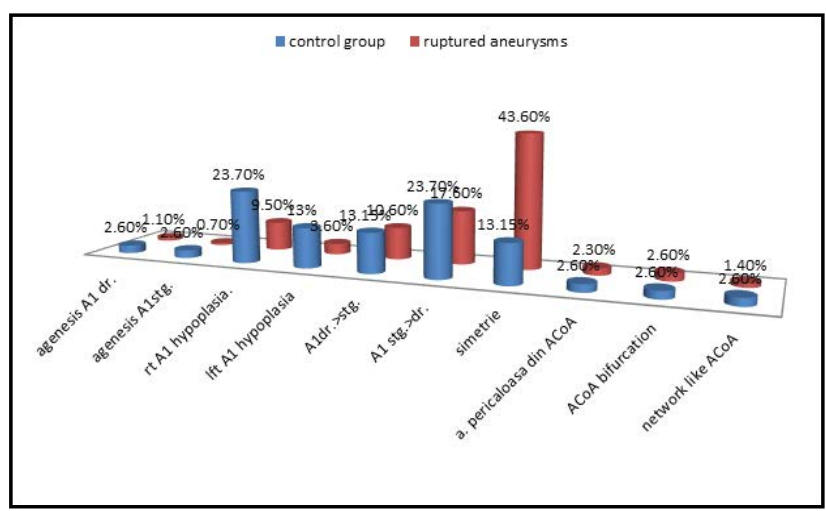

Figure 7-Anatomical anomalies distribution. 
Basically there is a clear dominance of asymmetric flux distribution between A1 segments. The anomalies that are affecting the anterior communicating artery are in equal percentage in control group and clinical group, cannot be considered as risk factor for rupture.

In the clinical group that included ruptured aneurysms, we calculated the ratio between the dominated vessel and the dominated vessel, excluding agenesis and hypoplasia, thus attempting to evaluate hemodynamic asymmetry and thus alteration of blood flow in ACoA, we obtained a ratio of 0.66 (Figure 8).

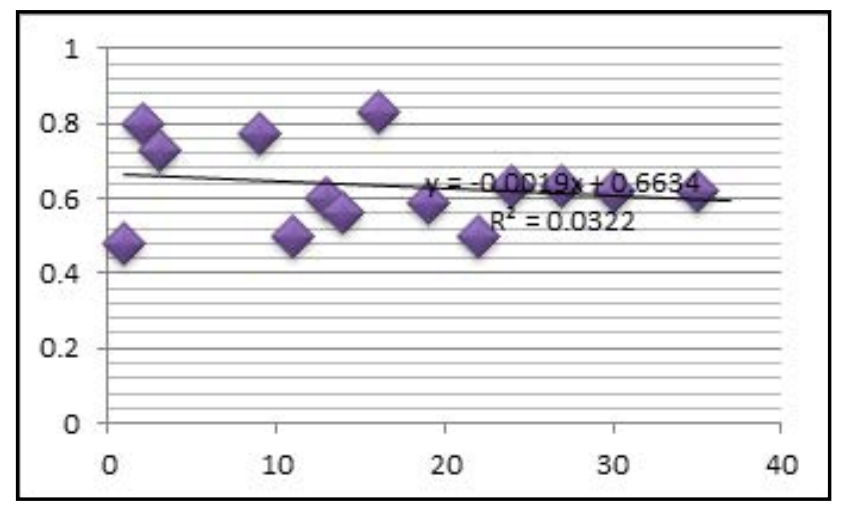

Figure 8-Average ratio A1 segment excluding malformation..

This value we propose as risk value in of both genesis and the risk of rupture of an aneurysm of anterior communicating artery.

Asymmetries in the distribution of local blood flow can cause stress and micro traumatisms on vascular walls, such an asymmetry of A1 segments gauge may pose a risk of aneurysm rupture.

The association between circle of Willis anomalies and aneurysm rupture in previous reports has been primarily descriptive or consisted of much fewer cases [(11,12].

\section{Conclusions}

The management of patients harboring unruptured intracranial aneurysms remains controversial and challenging because only a few, mostly demographic, risk factors for aneurysm rupture have been identified. This series aimed to study the use of more detailed anatomic information to assess rupture status. Our analysis suggests that ACoA aneurysms with ACA anomalies are associated with rupture.

According to our findings, we suggest another parameter to assess the risk of rupture of an aneurysm of the anterior communicating artery, i.e. the ratio of anterior cerebral artery A1 segment, which we consider to have the critical value of 0.65 and consider it to be an indication of aneurysm surgical cure.

Furtherstudy of the physiologic characterization of blood flow, flow patterns and fluid dynamics within different circle of Willis configurations and their role in aneurysm rupture is warranted.

\section{References}

1. Wiebers D.O., Whisnant J.P. \& Huston J 3rd, et al (2003). Unruptured intracranial aneurysms: natural history, clinical outcome, and risks of surgical and endovascular treatment. Lancet. 362, 103-10.

2. Bederson J.B., Awad I.A., Wiebers D.O. et al. (2000). Recommendations for the management of patients with unruptured intracranial aneurysms: a statement for healthcare professionals from the Stroke Council of the American Heart Association. Stroke. 39(1), 2742-50.

3. Rinkel G.J., Djibuti M. \& Algra A. et al. (1998). Prevalence and risk of rupture of intracranial aneurysms: a systematic review. Stroke.29,251-6

4. Forget T.R. Jr, Benitez R. \& Veznedaroglu E. et al. (2001) - A review of size and location of 
ruptured intracranial aneurysms. Neurosurgery. 49, 1322-5

5. Kapoor K., Singh B. \& Dewan L.I. (2008) Variations in the configuration of the circle of Willis. Anat Sci Int. 83, 96-106.

6. Lazzaro M.A., Ouyang B. \& Chen M. (2011). The role of circle of Willis anomalies in cerebral aneurysm rupture. $J$ NeuroIntervent Surg. doi:10.1136/jnis.2010.004358

7. Tanaka H., Fujita N. \& Enoki T. et al. (2006). Relationship between variations in the circle of Willis and flow rates in internal carotid and basilar arteries determined by means of magnetic resonance imaging with semiautomated lumen segmentation: reference data from 125 healthy volunteers. AJNR Am J Neuroradiol. 27, 1770-5.

8. Ingebrigtsen T., Morgan M.K. \& Faulder K. et al. (2004) - Bifurcation geometry and the presence of cerebral artery aneurysms. J Neurosurg. 101, 108-13.
9. Rhoton A.L. Jr. (2002). Aneurysms. Neurosurgery. 51(4 Suppl), S121-58.

10. Bor A.S., Velthuis B.K. \& Majoie C.B. et al. (2008). Configuration of intracranial arteries and development of aneurysms: a follow-up study. Neurology. 70, 700-5.

11. de Rooij N.K., Velthuis B.K. \& Algra A. et al. (2009). Configuration of the circle of Willis, direction of flow, and shape of the aneurysm as risk factors for rupture of intracranial aneurysms. J Neurol. 256, 45-50.

12. Stojanovic N., Stefanovic I. \& Randjelovic S. et al. (2009). Presence of anatomical variations of the circle of Willis in patients undergoing surgical treatment for ruptured intracranial aneurysms. Vojnosanit Pregl. 66, 711-17 